\title{
BÀI TOÁN XỬ LÝ ĐƯỜNG BREAKLINE TRÊN MÔ HÌNH TIN, SỬ DỤNG CẤU TRÚC DCEL
}

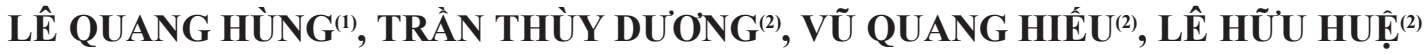 \\ ${ }^{(1)}$ Công ty Cổ phần Công nghệ Tài nguyên Môi trường và Vật liệu
}

${ }^{(2)}$ Truoòng Đại học Mỏ Địa chất

\section{Tóm tắt:}

Đường Breakline thuoòng mô tả là các đối tượng biểu diê̂n trên mô hình số địa hình nhu sống núi, khe núi, đồng mức.... Tuy nhiên, khi nghiên cưu bản đồ địa chính, cũng có thể ưng dụng nguyên tắc xủ lý đường Breakline để giải quyết một số bài toán địa chính trên mô hình số. Đối tượng nội dung chính của bản đồ địa chính gồm thưa đất và các đối tuợng địa lý liên quan nhu hệ thống giao thông, thủy hệ... Mô hình TIN địa chính được xây dưng tù tam giác hóa dĩ liệu đo đạc thực địa. Các cạnh thưa đất khi đura vào mô hình mặc nhiên là các cạnh cố định và thỏa mãn điều kiện cạnh của mạng lưới tam giác không được cắt qua. Nhu vậy, mạng lưới tam giác cần phải chỉnh sửa, biên tập để xư lý giao cắt. Cấu trúc dũ liệu DCEL có nhũng uu điểm trong luu trũ dũ liệu được lựa chọn để giải quyết vấn đề này phù hợp với thuật toán đề ra. Bài báo dùng hai phương pháp "Chia cạnh" và "Hoán vị” tam giác áp dụng xủ lý đường Breakline. Bài toán này là bài toán đi truócc, tạo tiền đề để giải quyết tiếp vấn đề tạo Topology cho đối tương vùng trên bản đồ địa chính ứng dụng mô hình TIN.

\section{1. Đặt vấn đề}

Hiện nay, khi mô tả bề mặt địa hình,mô hình TIN thường sử dụng. Một trong những thao tác khi biên tập cơ bản, quan trọng trên mô hình TIN là xử lý các đứt gãy địa hình, xử lý đường Breakline. Đường Breakline thường được dùng để mô tả sự thay đổi đột ngột hay còn gọi là các đặc trưng địa hình như: đường tụ thủy, đường phân thủy... Khi tam giác hóa dữ liệu địa hình, các cạnh tam giác có thể giao cắt với đường Breakline. Bài toán xử lý nhằm mục tiêu chỉnh sửa mô hình tam giác loại bỏ giao cắt và đường Breakline sẽ bị chia ra, ép buộc trở thành các cạnh của mô hình tam giác.Có rất nhiều phương pháp được ứng dụng để xử lý như: hoán đổi tam giác, chia cạnh...Sau xử lý, đường Breakline trên mô hình có thể được giữ nguyên hoặc bị phân chia và cấu trúc dữ liệu sẽ bị biến đổi. Các cạnh mới, các tam giác mới có thể được tạo thêm đòi hỏi phải cập nhật dữ liệu và xây dựng lại quan hệ tam giác. Cấu trúc DCEL tuycó những nhược điểm như tốn bộ nhớ, các tam giác biểu diễn ở dạng không tường minhnhưng lại có ưu điểm là rất linh hoạt khi thao tác biên tập trên mô hình TIN. Thực tế cho thấy, cấu trúc biểu diễn mô hình tam giác có ảnh hưởng rất lớn đến độ phức tạp của thuật toán cũng như là tốc độ của một thao tác cụ thể.Việc lựa chọn cấu trúc biểu diễn ngoài ý nghĩa thuận tiện cho lưu trữ, cập nhật dữ liệu còn phụ thuộc vào mục đích sử dụng sau này[2]. Trong trường hợp cụ thể của nghiên cứu này, với những đặc tính ưu việt, cấu trúc DCEL được lựa chọn để ứng dụng để xử lý đường Breakline.

Khi xử lý các bài toán địa chính trên mô hình TIN, các đối tượng dạng tuyến như cạnh thửa đất, cạnh nhà, đường quy hoạch, đường địa giới... có thể được xem là các đường Breakline. Lưới tam giác được xây dựng cũng có thể xảy ra trường hợp các cạnh tam giác cắt các đường Breakline. Cách giải quyết xử lý đường Breakline trong địa chính cũng tương tự như xử lý trong địa hình. Tuy nhiên, trong xử lý giữa địa chính và địa hình cũng có đặc điểm riêng. Trong 
dữ liệu địa hình, mô hình tam giác sau khi xử lý, các tam giác thu được thường phải kiểm tra và thỏa mãn điều kiện Delaunay. Trong địa chính, các tam giác được xây dựng chỉ cần phủ kín bề mặt cần mô tả, có thể tồn tại những tam giác bẹt, không nhất thiết phải thỏa mãn điều kiện tam giác Delaunay như trong địa hình[5]. Sử dụng mô hình TIN Delaunay xử lý đường Breakline trong địa chính là sự kế thừa và tận dụng kết quả của các thuật toán xây dựng mô hình tam giác Delaunayđã được nghiên cứu[4]. Mặt khác, sử dụng mô hình TIN Delaunay còn nhằm mục đích sử dụng cùng một mô hình dữ liệu cho xử lý kết hợp bài toán địa hình và địa chính từ một cơ sở dữ liệu chung.Nội dung bài báo sẽđưa ra một hướng giải quyết mớixử lý đường Breakline trên mô hình TIN, sử dụng cấu trúc DCEL có thể áp dụng cho bài toán địa hình, địa chính.

\section{Giải quyết vấn đề}

Giả sử người ta đưa vào một tập hợp điểm, sau đó tiến hành tam giác hóa, xây dựng mô hình dữ liệu. Trong tập hợp các điểm đó, có thể phân loại dữ liệu điểm thành các loại:

-Điểm có tính chất địa chính,

-Điểm có tính chất địa hình,

-Điểm có tính chất vừa là địa hình, vừa là địa chính (điểm địa chính có độ cao).

Trên cơ sở tập hợp điểm chung đó, dữ liệu địa hình đưa vào tập hợp dữ liệu địa hình, dữ liệu địa chính đưa vào tập hợp dữ liệu địa chính còn dữ liệu địa chính có độ cao được phân vào cả hai tập hợp trên. Mỗi tập hợp dữ liệu tùy thuộc vào mục đích mà sử dụng dữ liệu địa chính hay địa hình mà áp dụng các phương pháp giải quyết khác nhau. Nội dung sau đây sẽ nghiên cứu các hướng giải quyết áp dụng cho việc xử lý dữ liệu địa chính.

Mục tiêu nghiên cứu xử lý đường Breakline trên mô hình TIN là biến đổi sao cho cạnh Breakline sẽ phải biến thành các cạnh tam giác trên mô hình tam giác. Sau biến đồi mô hình tam giác sẽ bị chỉnh sửa, thay đổi, có thể thêm mới các đỉnh, tam giác nhưng cũng có thể chỉ hoán vị giữa hai tam giác liền kề nhau và dữ liệu phải được thay đổi lại (thêm mới hoặc cập nhật).

Việc cập nhật và thêm mới trong cấu trúc DCEL tiến hành theo 2 bước như sau:

Bước 1: Tìm tập hợp các nưa canh DCEL bi đuờng Breakline cắt qua và tập hợp các giao điểm

Bước 2: Trong tập hợp các nửa cạnh bị cắt vìa tìm được, xừ lý lần lượt các nưa cạh cắt theo tu tuờng cập nhật và thêm mói

Cấu trúc DCEL tổng quát được mô tả bằng ngông ngữ lập trình Visual Basic như sau:

e as long (số hiệu của nửa cạnh)

\section{Private Type EdgeDC}

vas long (đỉnh xuất phát của nưa cạh e)

$e_{t}$ as long (nủa cạh ngược của nủa cạh e)

$e_{n}$ as long (nưa cạnh tiếp theo của nưa cạn e)

T as long (tam giác chúa nưa cạh)

\section{End Type}

Ngoài ra còn có:

$e_{n n}$ as long (nưa cạnh tiếp của nưa cạnh tiếp của nứa cạnh e).

Trong nghiên cứu của bài báo này,nội dung xử lý đường Breakline sử dụng cấu trúc DCEL được giải quyết theo hai phương pháp:

- Phương pháp chia cạnh

- Phương pháp hoán đổi tam giác (Bảo toàn cạnh).

\subsection{Phuơng pháp “Chia cạnh"}

Có 2 cách thực hiện:

- Cách 1: Giữ được hình dạng tam giác có xu hướng đều (sau xử lý đường Breakline, các tam giác mới xây dựng sẽ được kiểm tra điều kiện Delaunay).

Cách xử lý này sẽ giữ cho những tam giác mới sau khi chia có xu hướng không bị bẹt bằng 
cách sau khi xử lý chia cạnh và thêm mới các đỉnh, các tam giác mới được xây dựng sẽ phải kiểm tra và thỏa mãn điều kiện Delaunay[5].

- Cách 2: Không quan tâm đến hình dạng tam giác (chia cạnh và xây dựng mới tam giác). Cách xử lý này có lợi thế là đơn giản, ổn định và không phải kiểm tra điều kiện tam giác. Tuy nhiên, do không kiểm tra điều kiện Delaunay nên sau xử lý có thể tạo ra những tam giác bẹt. Do vậy phải tùy điều kiện và mục tiêu cụ thể để áp dụng.

Theo phương pháp này, cách 1 phù hợp áp dụng xử lý Breakline trong các bài toán địa hình. Trong địa chính có thể áp dụng cách 2 (chia cạnh) trong xử lý các trường hợp như: chia tách thửa, tính toán giải phóng mặt bằng, xây dựng bản đồ quy hoạch...

Theo nguyên tắc của phương pháp, khi xét phải lần lượt đi theo các tam giác chứa các nửa cạnh có giao dọc theo đường Breakline. Ở các điểm giao cần phải thiết lập các đỉnh mới bằng các thao tác chèn điểm - thêm điểm vào mô hình tam giác. Các cạnh mới, các tam giác mới được thiết lập. Mô hình tam giác sẽ bị biến đổi và phải cập nhật lại trong dữ liệu các thành phần: $\mathrm{v}, \mathrm{e}_{\mathrm{t}}$, $\mathrm{e}_{\mathrm{n}}$, $\mathrm{T}$ của các nửa cạnh tam giác; đồng thời, thêm mới các nửa cạnh bị chia vào danh sách cạnh DCEL.

Việc chia cạnh, thêm mới và cập nhật lại trong danh sách cạnh DCEL được biểu diễn cụ thể qua hình và bảng sau: (Xem hình 1,2$)$

\section{Các bước thục hiện:}

Bước 1: Tìm các nửa cạnh giao với đường Breakline (cạnh màu đỏ, hình dưới) trong tập hợp các cạnh DCEL ban đầu:

-Tìm nửa cạnh có đỉnh gốc là điểm đầu của đường Breakline.

Trong cấu trúc DCEL, đỉnh gốc của cạnh e chính là đỉnh đích của cạnh $\mathrm{e}_{\mathrm{nn}}$ và cũng chính là điểm khởi đầu của đường Breakline. Do vậy, chỉ cần xét xem nửa cạnh $\mathrm{e}_{\mathrm{n}}$ có giao với Breakline hay không.

Sư dụng hàm Intersection để xét giao của nưa cạnh $e_{n}$ với Breakline và xét:

Nếu có giao thì thêm nứa cạnh $e_{n}$ vì̀a tìm

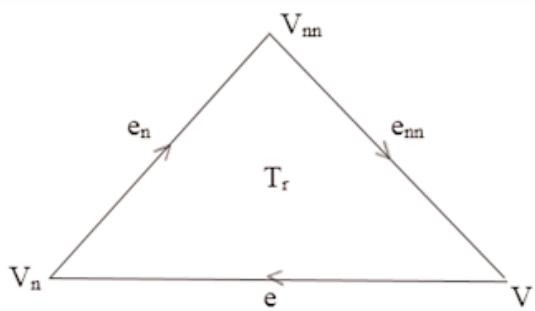

\begin{tabular}{|c|c|c|c|c|}
\hline $\mathbf{e}$ & $\mathbf{V}$ & $\mathbf{e}_{\mathbf{t}}$ & $\mathbf{e}_{\mathbf{n}}$ & $\mathbf{T}_{\mathbf{r}}$ \\
\hline $\mathrm{e}$ & $\mathrm{V}$ & $\mathrm{e}_{\mathrm{t}}$ & $\mathrm{e}_{\mathrm{n}}$ & $\mathrm{T}_{\mathrm{r}}$ \\
\hline $\mathrm{e}_{\mathrm{n}}$ & $\mathrm{V}_{\mathrm{n}}$ & $\mathrm{e}_{\mathrm{tn}}$ & $\mathrm{e}_{\mathrm{nn}}$ & $\mathrm{T}_{\mathrm{r}}$ \\
\hline $\mathrm{e}_{\mathrm{nn}}$ & $\mathrm{V}_{\mathrm{nn}}$ & $\mathrm{e}_{\mathrm{tnn}}$ & $\mathrm{e}$ & $\mathrm{T}_{\mathrm{r}}$ \\
\hline
\end{tabular}

Hình 1: Dũ liệu trước khi chia cạnh

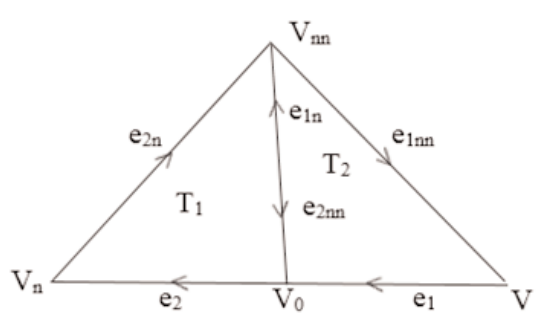

Ký hiệu: --- Không thay đồi
\begin{tabular}{|c|c|c|c|c|}
\hline $\mathbf{e}$ & $\mathbf{V}$ & $\mathbf{e}_{\mathbf{t}}$ & $\mathbf{e}_{\mathbf{n}}$ & $\mathbf{T}_{\mathbf{r}}$ \\
\hline $\mathrm{e}_{1}$ & --- & & $\mathrm{e}_{1 \mathrm{n}}$ & $\mathrm{T}_{1}$ \\
\hline $\mathrm{e}_{1 \mathrm{n}}$ & $\mathrm{V}_{0}$ & $\mathrm{e}_{2 \mathrm{nn}}$ & $\mathrm{e}_{1 \mathrm{nn}}$ & $\mathrm{T}_{1}$ \\
\hline $\mathrm{e}_{1 \mathrm{nn}}$ & --- & --- & $\mathrm{e}_{1}$ & $\mathrm{~T}_{1}$ \\
\hline $\mathrm{e}_{2}$ & $\mathrm{~V}_{0}$ & & $\mathrm{e}_{2 \mathrm{n}}$ & $\mathrm{T}_{2}$ \\
\hline $\mathrm{e}_{2 \mathrm{n}}$ & --- & --- & $\mathrm{e}_{2 \mathrm{nn}}$ & $\mathrm{T}_{2}$ \\
\hline $\mathrm{e}_{2 \mathrm{nn}}$ & $\mathrm{V}_{\mathrm{nn}}$ & $\mathrm{e}_{1 \mathrm{n}}$ & $\mathrm{e}_{2}$ & $\mathrm{~T}_{2}$ \\
\hline
\end{tabular}

Hình 2: Dũ liệu sau khi chia cạnh 
được vào một mảng tạm. Gán $e=e_{n}$ và thoát vòng lạp.

Nếu không giao, lấy nưa cạnh đảo $e_{t}$ của e vàgán $e=e_{t}$. Thực hiện vòng lặp để xét cạnh giao tương tư với nưa canh e mói vùa gán.

Thực hiện thao tác lặp lại cho tới khi tìm được nưa cạh giao đầu tiên.

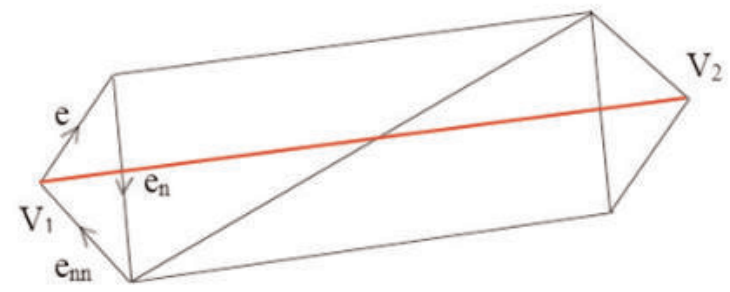

Hình 3: Tìm nưa cạnh giao đầu tiên $\left(e_{n}\right)$

- Sau khi đã tìm được nửa cạnh giao đầu tiên, sử dụng chính nửa cạnh giao đó để gán làm cạnh e tiếp theo thực hiện vòng lặp.Tuy nhiên, lúc này cần phải xét cả $e_{n}$ và $e_{n n}$ xem có giao với Breakline hay không bởi vì đỉnh gốc của nửa cạnh e đang xét lúc này không còn trùng với điểm đầu của Breakline.

- Thực hiện vòng lặp để xét, cứ mỗi khi xác định được cạnh giao thì cạnh giao đó được thêm vào mảng tạm. Sau đó, sử dụng cạnh giao vừa tìm được gán làm nửa cạnh e và thực hiện lại thao tác lặp tìm kiếm cạnh giao.

Kết quả cuối cùng thu được một mảng tạm gồm các nửa cạnh giao với đường Breaklline.

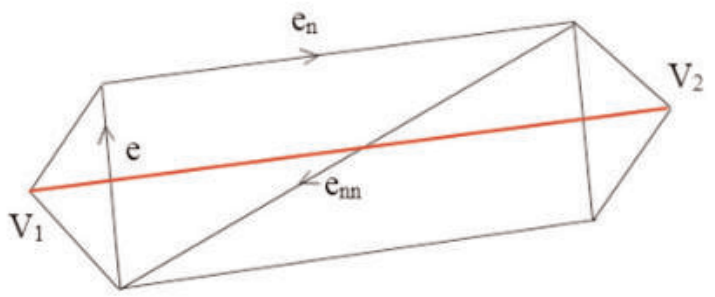

Hình 4: Tìm cạnh giao tiếp theo

Bước 2: Tìm tọa độ điểm giao và thêm mới vào danh sách điểm.

-Duyệt trong mảng danh sách các nửa cạnh giao vừa tìm được, dùng hàm tìm giao điểm để tìm lần lượt các điểm giao với Breakline của từng nửa cạnh.

-Thêm các điểm giao vừa tìm được vào danh sách điểm.

Bước 3: Chèn điểm các điểm giao vừa tìm được vào mô hình tam giác, thêm mới và cập nhật lại trong cấu trúc dữ liệu.

\section{Kết thúc thực hiện}

Nhận xét:

Hạn chế của phuoong pháp xủ lý đường Breakline theo nguyên tắc "Chia cạnh"là tạo ra môt số luợng lớn các đỉnh thêm và các cạnh thêm không mong muốn. Tuy nhiên, nếu áp dụng nguyên tắc này trong một số bài toán địa chính lại có ưu điểmnhu khi tách thửa, hợp thửa, quy hoạch sủ dụng đất, giải phóng mặt bằng...Khi xủ lý Breakline trong các bài toán địa chính, không cần phải kiểm tra điều kiện Delaunay bởi vì việc tạo ra các tam giác bẹt trong quá trình xủ lý không làm ảnh hương đến độ chính xác hình thể cũng như diện tích của thữa đất.

\subsection{Phương pháp "Hoán đổi” tam giác}

Phương pháp "Hoán đổi” tam giác còn gọi là Flip tam giác vàcó thể hiểu là phương pháp bảo toàn cạnh. Sau khi đã xác định được các cạnh của mô hình tam giác giao với đường Breakline, tiến hành lần lượt hoán đổi tam giác theo từng cặp tam giác liền kề có cạnh chung, giao cắt với đường Breakline. Phương pháp này sử dụng thao tác Flip tam giác đã được trình bày trong [3]. Khi đó, dữ liệu các tam giác sau hoán đổi sẽ phải được cập nhật lại trong cấu trúc dữ liệu DCEL

\section{Các bước thục hiện}

Buớc 1: Tìm các nưa canh giao với đường Breakline trong tập hơp các cạnh DCEL ban đầu.

Ở bước này, thao tác duyệt và tìm các nưa cạnh giao vói Breakline tuoong tụ nhu phwơng pháp chia cạh. Các nưa cạnh giao sẽ được thêm vào mảng tạm. 
Bước 2: Duyệt trong mảng tạm chúa các nưa cạnh giao với Breakline, kiểm tra xem điều kiệnFlip:

-Nếu thỏa mãn điều kiện thì tiến hành thao tác hoán đổi tam giác (Flip). Sau đó tiếp tục kiểm tra nưa cạnh đó sau khi Flip có còn cắt Breakline hay không. Nếu không còn cắt nũa thi loại nưa cạnh đó khỏi mảng tạm chúa các nưa cạnh giao.

-Nếu còn cắt thì giữ lại và tiếp tục thực hiện vòng lặp.

-Nếu không thỏa mãn thì tiếp tục duyệt tiếp cạnh tiếp theo.

-Dùng lại khi không còn cạnh nào trong danh sách mảng tạm

Bước 3: Đánh dấu các nưa cạnh Breakine sau khi xư lý đã biến đổi thành cạn tam giác.

-Sư dụng một biến đánh dấu.

-Gán giá trị biếnbằng -1 cho các nưa cạnh đã được xủ lý và trở thành cạnh tam giác trước khi thựchiện thao tác loại cạnh đó khỏi mảng chưa các nứa canh giao.

(Thao tác này nhằm mục đích để đánh dấu đường Breakline nhưng cũng đồng thời là cạnh cố định - cạnh thửa đất để sủ dụng trong bài toán tạo Topologycho thưa đất trên bản đồ địa chính).

\section{Nhận xét:}

Uu điểm của phương pháp "Hoán đổi" tam giác là sau xủ lý, đuờng Breakline được giư nguyên (bảo toàn) không thay đổi. Quá trình xủ lý không tạo ra các đỉnh mới và các tam giác mói trong mô hình nên hạn chế tăng thêm các phép tính toán.

Nhược điểm của phurơng pháp là có thể phải thực hiện vòng lạp nhiều lần cho tới khi loại được hết các cạnh giao cắt với đường Breakline. Việc thực hiện lạ̣p đi lạp lại một thao tác với một số điểm lớn sẽ làm chậm chưong trình.

\section{Thực nghiệm}

Số liệu đưa vào thực nghiệm là số liệu giả định. Các điểm đo vừa có tính chất địa hình, vừa có tính chất địa chính.

Trong trường hợp dữ liệu điểm đo thuần địa chính hoặc thuần địa hình (theo phân loại trong nội dung trình bày ở phần lý thuyết) không làm ảnh hưởng đến thuật toán của phương pháp xử lý đã trình bày. Điều khác biệt là việc xử lý đó phục vụ mục tiêu nào, giải quyết bài toán địa chính hay bài toán địa hình mà thôi.

\subsection{Phương pháp chia cạnh}

Giả sử mô hình tam giác với dữ liệu như sau:

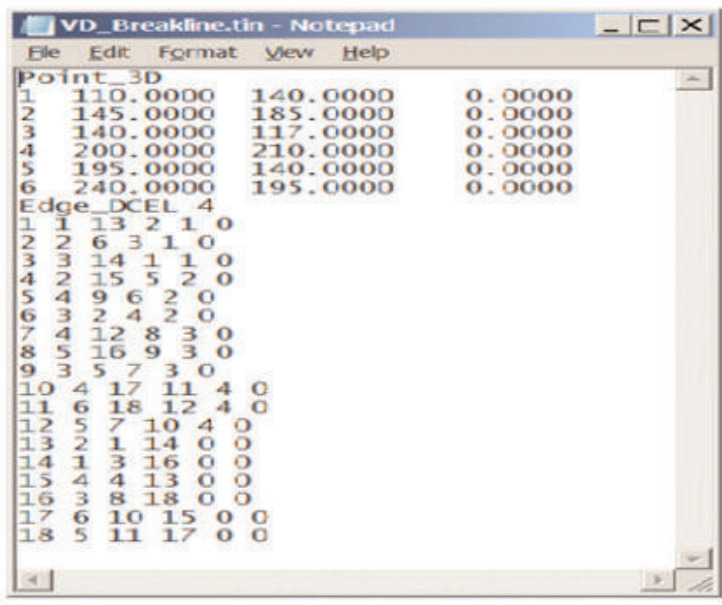

Hình 5: Dũ liệu đầu vào ban đầu

Bước 1: Tam giác hóa và chuyển về DCEL.

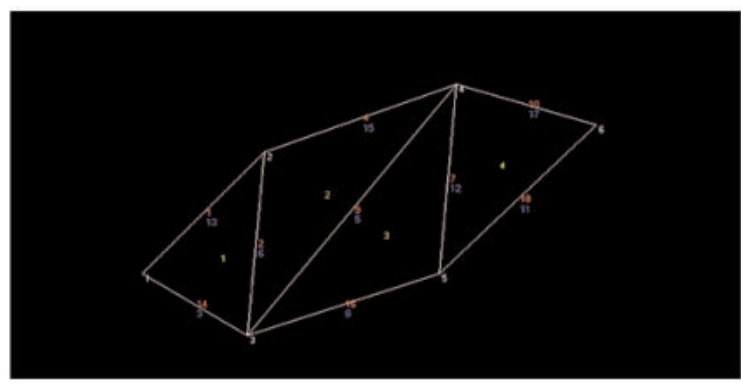

Hình 6: Tam giác hóa dũ liệu và chuyển đổi cấu trúc DCEL

Bước 2: Chèn đường Breakline $(1$ - 6) vào mạng lưới tam giác. 


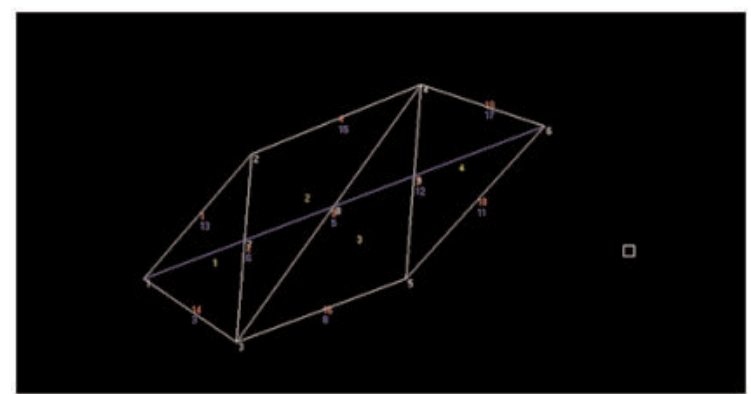

Hình 7: Đua đưòng Breakline vào mang lưới tam giác

Bước 3: Chia đường Breakline bằng các điểm nội suy và cập nhật lại danh sách các cạnh DCEL.

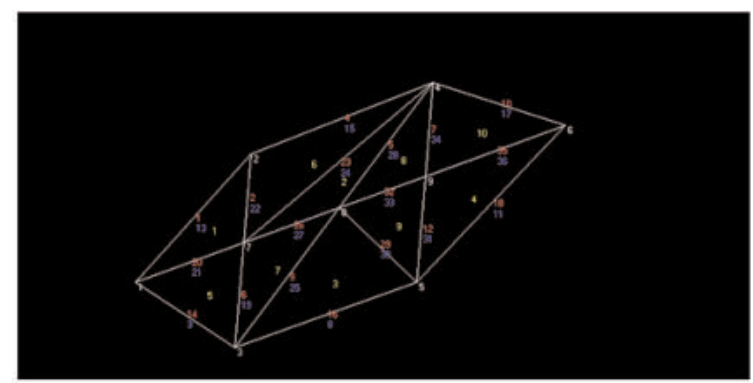

Hình 8: Tìm các điểm giao, tạo tam giác mói và cập nhật dũ liệu DCEL

\subsection{Thưc nghiệm phương pháp "Hoán đổi"} hay Flip tam giác

Bước 1: Tam giác hóa xây dựng mô hình TIN, chuyển về DCEL và đưa cạnh Breakline (16) vào mô hình tam giác.

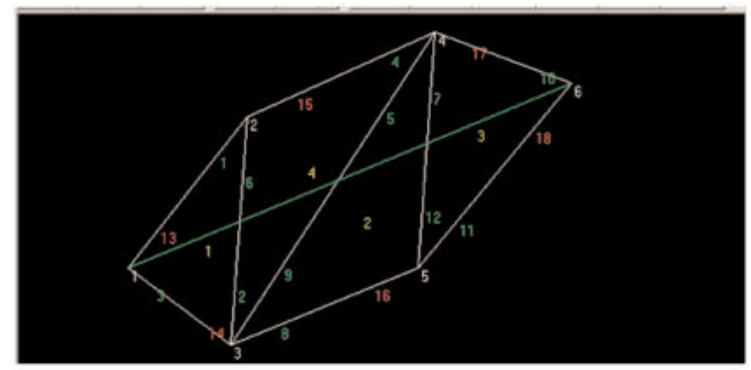

Hình 9: Xây dưng lưới tam giác, chuyển về DCEL, dura cạh Breakline vào

Bước 2: "Hoán đổi" cho cặp tam giác liền kề 1 và 2 và cập nhật lại dữ liệu DCEL.

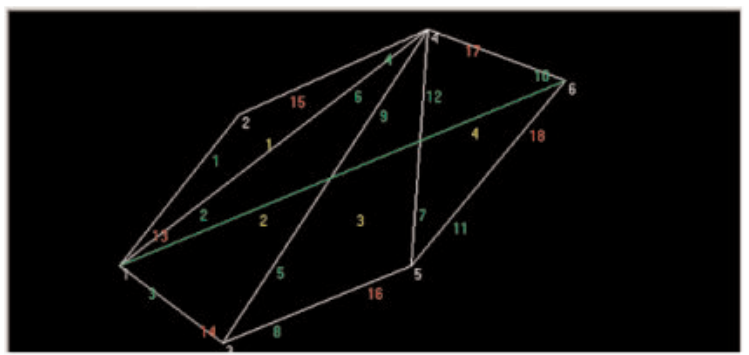

Hìn 10: Hoán đổi cặp tam giác 1,2

Bước 3: "Hoán đổi" cho cặp tam giác liền kề 2 và 3 và cập nhật lại dữ liệu DCEL.

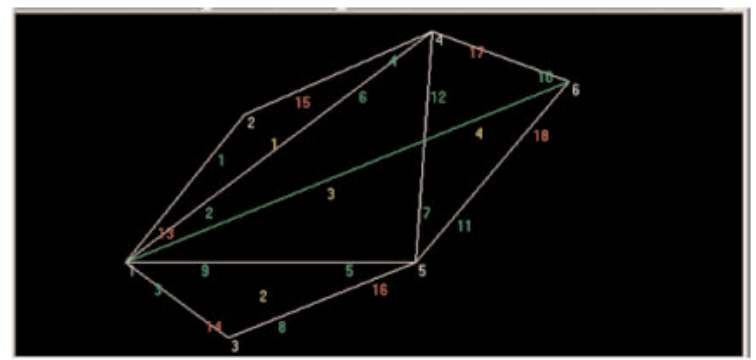

Hình 11: Hoán đổi cặp tam giác 2,3

Bước 4: "Hoán đổi” cho cặp tam giác liền kề 3 và 4 và cập nhật lại dữ liệu DCEL.

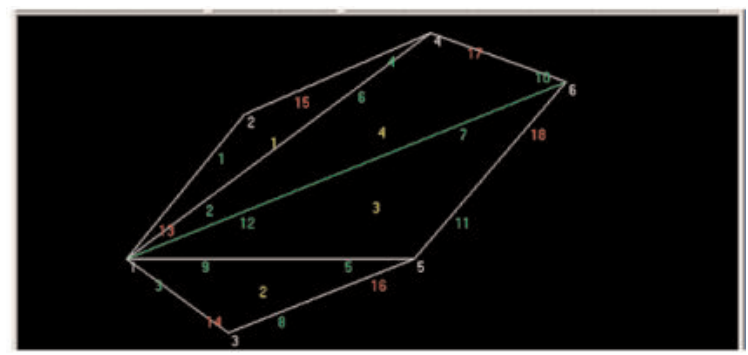

Hình 12: Hoán đổi cặp tam giác 3,4

\section{Kết luận}

- Xử lý đường Breakline theo nguyên tắc "Chia cạnh" phù hợp với thao tác trên mô hình TIN địa hình (khi bắt buộc phải thỏa mãn điều kiện Delaunay). U’u điểm của phương pháp là tam giác mới tạo ra sẽ có xu hướng đều, tương đồng với lưới tam giác ban đầu, thuận lợi cho các bài toán nội suy địa hình. Một ưu điểm nữa của phương pháp khiáp dụng trong các bài toán chia tách thửa đất, quy hoạch, giải phóng mặt bằng ... trên mô hình TIN địa chính thì không cần kiểm tra điều kiện Delaunay. 
- Xử lý đường Breakline theo nguyên tắc "Hoán đổi tam giác" có ưu điểm cạnh Breakline sau xử lý được bảo toàn nguyên vẹn. Tuy nhiên, xử lý theo các nguyên tắc này có thể sẽ tạo ra những tam giác bẹt. Trong địa chính vấn đề xử lý đường Breakline nhằm loại bỏ các giao cắt của cạnh tam giác với cạnh thửa đất. Cho nên, trong mô hình tam giác tồn tại các tam giác bẹt không làm ảnh hưởng đến quan hệ Topologygiữa các thửa đất.

- Nếu ta coi các đường Breakline là các đường cạnh thửa, đường quy hoạch, đường chỉ giới thì cả hai phương pháp xử lý đường Breakline đều phù hợp áp dụng trong việc giải quyết các bài toán địa chính. Từ đó, cùng với sự linh hoạt trong xử lý và những ưu điểm trong các thao tác biên tập của cấu trúc DCEL, ta có thể áp dụng giải quyết kết hợp cả hai dữ liệu địa chính và địa hình từ một cơ sở dữ liệu chung.

- Ngoài ra, với tính cục bộ của cấu trúc DCEL, việc xử lý Breakline còn là cơ sở để bước đầu giải quết các bài toán chồng phủ [1], cập nhật biến động, tạo vùng... trong quản lý đất đai. $\bigcirc$

\section{Tài liệu tham khảo}

\section{Summary}

\section{Problem solving Breakline line on TIN model, using Dcel structure}

\section{Le Quang Hung, Resource Enviroment and Materials Technology Joinstock Company}

Tran Thuy Duong, Vu Quang Hieu, Le Huu Hue, University of Mining and Geology

Breakline lines are often described as objects that perform on topographic models such as mountain, ravine, level... However, when studying cadastral maps, it is also possible to apply Breakline road handling principles to solve some cadastral problems on the numerical model. The main content objects of the cadastral map include land plots and related geographic features such as transport systems, hydrology... The cadastral TIN model is built from the triangle of field measurements. The parcel of land when included in the model is automatically fixed edges and satisfy the condition of the edge of the triangular network is not cut. Thus, triangular networks need to edit and edit to handle intersections. DCEL data structure has advantages in data storage chosen to solve this problem in accordance with the proposed algorithm. The paper uses two methods of "Split edge" and "Diagram" of triangle applied Breakline processing. This problem is a preceding problem, creating a premise to solve the problem of creating Topology for regional objects on the cadastral map application model. $\bigcirc$ 